\title{
Reviews of "Neurological manifestations associated with COVID-19: a nationwide registry"
}

\section{Yi Tang ${ }^{1}$, Chenjian Li ${ }^{2}$, Kapil Gururangan ${ }^{3}$}

${ }^{1}$ Professor in Neurology, Department of Neurology, Xuanwu Hospital, Capital Medical University, National Clinical Research Center for Geriatric Diseases Beijing, China,

${ }^{2}$ Professor, School of Life Sciences, Peking University, Beijing, China,

${ }^{3}$ Department of Neurology, Icahn School of Medicine at Mount Sinai

Published on: Jan 11, 2021

DOI: $10.1162 / 2 \mathrm{e} 3983 f 5 . c 908508 \mathrm{c}$

License: Creative Commons Attribution 4.0 International License (CC-BY 4.0). 
To read the original manuscript, click the link above.

Summary of Reviews: The study finds broad spectrum of neurological manifestations associated with SARS-CoV-2 infection. Findings are informative for future intervention studies. Decision-makers should consider the claims in this study actionable with limitations to some methods and data.

\section{Reviewer 1 (Yi Tang, Chenjian Li) | |}

Reviewer 2 (Kapil Gururangan) |

$$
\begin{aligned}
& \text { RR:C19 Strength of Evidence Scale Key } \\
& \text { प्रमप = Misleading } \\
& \text { प्र००० = Not Informative } \\
& \text { प्रा पि = Potentially Informative }
\end{aligned}
$$

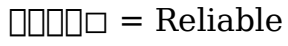

$$
\begin{aligned}
& \text { प्राप्र = Strong }
\end{aligned}
$$

To read the reviews, click the links below. 